\title{
Nível de atividade física, qualidade do sono e de vida em pacientes com doença arterial periférica
}

\author{
Level of physical activity, quality of sleep and life in patients with peripheral arterial \\ disease
}

Ariel Fukushima Fallaci ${ }^{1}$, Daiana de Jesus da Silva Mendes², João Victor Silva dos Santos ${ }^{3}$, Alice Maria Borges de Carvalho ${ }^{4}$, Nilo Manoel Pereira Vieira Barreto ${ }^{5}$, Michelli Christina Magalhães Novais $6^{6 *}$

${ }^{1}$ Fisioterapeuta pela Faculdade Metropolitana de Camaçari (FAMEC-BA); ${ }^{2}$ Acadêmica do Curso de Fisioterapia da FAMEC-BA; ${ }^{3}$ Acadêmico do Curso de Enfermagem da FAMEC-BA; ${ }^{4}$ Fisioterapeuta pela Faculdade Adventista de Fisioterapia (FAFIS), Especialista em Micropolítica da Gestão e do Trabalho em Saúde pela Universidade Federal Fluminense (UFF-RJ), Fisioterapeuta do Hospital Ana Nery-BA. ${ }^{5}$ Enfermeiro pela Universidade Católica de Goiás (PUC-GO), Mestre e Doutorando pelo Programa de Pós-graduação em Processos Interativos dos Órgãos e Sistemas da Universidade Federal da Bahia (UFBA); ${ }^{6}$ Fisioterapeuta pela Faculdade Social da Bahia (FSBA), Mestre e Doutoranda do Programa de Pós-graduação em Processos Interativos dos Órgãos e Sistemas (UFBA), Professora no Centro Universitário Jorge Amado (UNIJORGE-BA)

\begin{abstract}
Resumo
Introdução: a Doença Arterial Periférica (DAP) decorre do estreitamento ou oclusão arterial, que interfere no aporte sanguíneo das extremidades inferiores. A DAP pode levar a um repouso prolongado, causando prejuízos à qualidade de vida e do sono dos pacientes, devido à dor e receio de lesão. Objetivo: descrever o nível de atividade física, a qualidade de vida e do sono em pacientes com DAP. Metodologia: trata-se de um estudo observacional, epidemiológico e transversal, realizado no Hospital Geral de Camaçari-BA. Foram incluídos pacientes com diagnóstico de DAP, ambos os sexos, com idade superior a 18 anos, internados no referido hospital. Foram excluídos os pacientes com distúrbios psiquiátricos e dificuldade de compreensão dos questionários. Para a avaliação do nível de atividade física foi utilizado o Questionário Internacional de Atividade física, para Qualidade de Vida o questionário Short Form Health Survey 36, para qualidade do sono Índice de Qualidade do Sono de Pittsburgh e para Claudicação Intermitente o Questionário de Edimburgo. Resultados: dos 27 pacientes analisados, 55,6\% (15/27) eram do sexo feminino e 44,4\% (12/27) masculino, 37\% (10/27) de cor/raça preta. A média de idade foi de $62,6 \pm 8,3$ anos, peso $71,8 \pm 16,2 \mathrm{~kg}$, altura $164,8 \pm 8,3 \mathrm{~cm}$ e IMC $26,3 \pm 5$. A maioria relatou ser ativo, 33,3\% (9/27). Segundo a SF-36 o domínio mais limitante foi "dor" $(28,6)$, o escore global do PSQI foi de 10,4 e a maioria $(81,5 \%)$ não apresentava claudicação intermitente. Conclusão: a presença de DAP foi mais frequente nos idosos e foram identificados a presença de distúrbios do sono e diminuição da qualidade de vida.

Palavras-chave: Arteriopatias Oclusivas. Privação do Sono. Atividade Motora. Claudicação Intermitente.
\end{abstract}

\begin{abstract}
Introduction: peripheral Arterial Disease (PAD) is due to narrowing or arterial occlusion, which interferes with the blood supply of the lower extremities. PAD can lead to prolonged rest, causing damage to the quality of life and sleep of patients, due to pain and fear of injury. Objective: to describe the level of physical activity, quality of life and sleep in patients with PAD. Methodology: this is an observational, epidemiological and cross-sectional study, carried out at the Hospital Geral de Camaçari-BA. Patients diagnosed with PAD, both sexes, aged over 18 years, admitted to the hospital were included. Patients with psychiatric disorders and difficulty in understanding the questionnaires were excluded. To assess the level of physical activity, the International Physical Activity Questionnaire was used, for Quality of Life the Short Form Health Survey 36 questionnaire, for sleep quality Sleep Quality Index of Pittsburgh and for Intermittent Claudication the Edinburgh Questionnaire. Results: of the 27 patients analyzed, 55.6\% (15/27) were female and 44,4\% $(12 / 27)$ were male, $37 \%(10 / 27)$ were black / colored. The mean age was $62.6 \pm 8.3$ years, weight $71.8 \pm 16.2 \mathrm{~kg}$, height $164.8 \pm 8.3$ $\mathrm{cm}$ and BMI $26.3 \pm 5$. The majority reported being active, 33.3\% (9/27). According to SF-36, the most limiting domain was "pain" (28.6), the global PSQI score was 10.4 and the majority (81.5\%) did not have intermittent claudication. Conclusion: the presence of $P A D$ was more frequent in the elderly and the presence of sleep disorders and decreased quality of life was identified.

Keywords: Arterial Occlusive Diseases. Sleep Deprivation. Motor Activity. Intermittent Claudication.
\end{abstract}

Correspondente/Corresponding: *Michelli Christina Magalhães Novais - Instituto de Ciências da Saúde da Universidade Federal da Bahia - End: Av. Reitor Miguel Calmon s/ $\mathrm{n}$ - Vale do Canela, CEP 40.110-100 Salvador, BA - Tel: (71) 9 8727-4948 - E-mail: novaismichelli@outlook.com

\section{INTRODUÇÃO}

A Doença Arterial Periférica (DAP) é uma complicação progressiva, decorrente de estreitamento ou oclusão arterial, que interfere no aporte sanguíneo das extremidades inferiores (SHABANI VARAKI et al., 2018). As principais 
causas da DAP são a aterosclerose, diabetes, hipercolesterolemia e hipertensão (MUIR, 2009).

A incidência da DAP tem aumentado mundialmente. No ano 2017, foram verificados 10,8 milhões de casos novos e 118,1 milhões de casos prevalentes. Além disso, houve também um aumento no número de óbitos de $55,7 \%$ quando comparado ao ano de 2007 (JAMES et al., 2018; KENGNE; ECHOUFFO-TCHEUGUI, 2019). A doença está presente em aproximadamente $13 \%$ da população com idade superior a 50 anos (FARAH et al., 2014), sendo que os índices tendem a aumentar após os 70 anos de idade (ALVIM et al., 2018).

A DAP apresenta-se geralmente de forma assintomática, contudo, um sintoma clássico é a presença de Claudicação Intermitente (CI) (SHABANI VARAKI et al., 2018), que acomete cerca de $10 \%$ dos indivíduos com a doença (VIRANI et al., 2020). A Cl pode ser caracterizada por dor muscular ao exercício físico que tende a cessar ao repouso (BARBOSA; DALLE, 2008). Por conta da dor e do receio de gerar algum tipo de lesão, o paciente que apresenta $\mathrm{Cl}$ tende a se manter em repouso por um período prolongado, mesmo alguns trabalhos sugerindo que a prática de atividade física reduz o risco de mortalidade desta população (BARBOSA; DALLE, 2008).

Alterações no sono podem trazer prejuízos consideráveis para as funções e atividades do paciente com DAP. O sono é essencial para a vida humana, ele contribui para conservação de energia, proteção e restauração dos sistemas (ARAGÃO et al., 2018). As condições de saúde e inatividade física podem influenciar negativamente na qualidade do sono (BARROS et al., 2019). A DAP pode interferir no sono do paciente, principalmente devido à dor, e com isso causar repercussões negativas na rotina diária (WANN-HANSSON et al., 2005) e na qualidade de vida (WU et al., 2017).

Pacientes sedentários podem apresentar impactos biopsicossociais e redução na qualidade de vida (ARAGÃO et al., 2018). Esta pode ser compreendida como a percepção do indivíduo em relação aos aspectos físicos, sociais, ambientais, espirituais e psicológicos que o envolvem (FLECK, 2000). A DAP, por se tratar de uma doença crônica e limitante, pode comprometer significativamente a qualidade de vida, necessitando de estratégias multidisciplinares para a adaptação do indivíduo à sua doença, na tentativa de promover a melhora de seu bem-estar (VAZ et al., 2013).

Apesar das informações disponíveis na literatura acerca da DAP e suas repercussões, são necessários estudos que investiguem o nível de atividade física, a qualidade de vida e a qualidade do sono desta população, uma vez que a compreensão desses aspectos pode auxiliar na implementação de estratégias de prevenção e de tratamento mais eficazes para os indivíduos com DAP. Deste modo, o objetivo do presente estudo foi descrever o nível de atividade física, a qualidade de vida e do sono de pacientes com DAP.

\section{METODOLOGIA}

Trata-se de um estudo observacional, epidemiológico e transversal, realizado no Hospital Geral de Camaçari HGC, cidade de Camaçari, Bahia, Brasil. Obedecendo a resolução 466/12 do Conselho Nacional de Ética em Pesquisa envolvendo seres humanos, todos os participantes do estudo assinaram o Termo de Consentimento Livre e Esclarecido. Este trabalho foi aprovado pelo Comitê de Ética e Pesquisa do hospital Ana Nery, sob o parecer de número 2.979.096.

Foram incluídos pacientes com diagnóstico clínico DAP, de ambos os sexos, com idade igual ou superior a 18 anos, internados no referido hospital. Foram excluídos os pacientes com distúrbios psiquiátricos e dificuldade de compreensão dos questionários aplicados.

Dados primários foram utilizados, colhidos pelos pesquisadores por amostragem não-probabilística, entre outubro de 2018 a junho de 2019. Os dados foram obtidos através da aplicação de questionários e busca ativa de informações nos prontuários dos pacientes. As seguintes variáveis foram analisadas: idade, sexo, peso, altura, IMC, renda familiar, atividade laboral, escolaridade, comorbidades, nível de atividade física, presença de claudicação intermitente, qualidade do sono e qualidade de vida.

Para a avaliação do nível de atividade física foi utilizado o Questionário Internacional de Atividade Física (IPAQ), instrumento que permite estimar o tempo semanal gasto em atividades físicas em diferentes contextos do cotidiano, tais como trabalho, transporte, tarefas domésticas e lazer. Neste estudo foi utilizada a versão curta do IPAQ, que apresenta a mesma efetividade, porém com uma adesão maior dos pacientes. O IPAQ curto é composto por sete questões abertas que permitem estimar o tempo gasto, por semana, em diferentes situações, como caminhadas e esforços físicos de intensidades moderada e vigorosa e de inatividade física. O resultado do questionário classifica os pacientes em sedentários, irregularmente ativos $A$, que são aqueles que atingem pelo menos um dos critérios de frequência e/ou duração de atividade física, irregularmente ativos $B$, que são aqueles que não chegam a atingir o mínimo de frequência ou de duração dos critérios do questionário, ativos ou muito ativos (MATSUDO et al., 2001).

A análise da presença de Claudicação Intermitente foi realizada através do Questionário de Claudicação de Edimburgo, que é composto por 5 perguntas objetivas e uma imagem dos membros inferiores (vista anterior e posterior) para que o indivíduo aponte o local que sente dor ou desconforto. O resultado é classificado como positivo/presença de claudicação caso as respostas às perguntas tenham sido $1=$ "sim", 2= "não", 3= "sim", $5=$ "geralmente desaparece em 10 minutos ou menos" e 6= "panturrilha" e/ou "coxa" e/ou "região glútea" independentemente de terem sido assinalados outros lugares e negativo se qualquer combinação diferente da descrita acima for observada. A questão 4 não é utilizada 
para definir a presença e sim a gravidade da claudicação (MAKDISSE et al., 2007).

Para a análise da qualidade de vida utilizou-se o questionário Short Form Health Survey 36 (SF-36), cujas pontuações podem variar de 0 a 100. O SF-36 é uma ferramenta empregada visando identificar as diferenças clínicas e sociais consideráveis para a saúde, assim como as mudanças na saúde ao longo do tempo. O questionário SF-36 é composto por 36 perguntas, agrupadas em oito escalas ou domínios. As pontuações mais altas indicam um melhor estado de saúde (LAGUARDIA et al., 2013).

A avaliação da qualidade do sono foi realizada com o Índice de Qualidade do Sono de Pittsburgh (PSQI), que possibilita a avaliação do sono qualitativamente e quantitativamente, correspondente ao último mês, não permitindo avaliações de problemas existentes no momento. As questões do PSQI estão dispostas em sete domínios, aos quais podem ser atribuídos de zero a três pontos. A somatória total deste instrumento pode atingir 21 pontos, sendo que os escores superiores a 5 pontos indicam padrão ruim de qualidade de sono. Trata-se de um questionário fácil de ser respondido, capaz de diferenciar os pacientes entre "bons dormidores" e "maus dormidores" (PASCOTTO; SANTOS, 2013).

As informações colhidas através da aplicação dos questionários IPAQ, SF-36 e PSQI faziam referência ao passado do paciente, período em que não estavam internados no hospital.

Os dados foram armazenados no IBM SPSS software (versão 19.0 para Windows, Estados Unidos da América). As variáveis contínuas foram expressas em medidas de tendência central e dispersão, enquanto as variáveis categóricas frequência absoluta e relativa.

\section{RESULTADOS}

Foram triados 29 pacientes, destes, dois foram excluídos por não apresentarem condições psicológicas de responder aos questionários completamente. Dos 27 pacientes analisados, 55,6\% (15/27) eram do sexo feminino, 37\% (10/27) de cor/raça preta, 44,2\% (12/27) eram analfabetos, $51,9 \%(14 / 27)$ referiram ser casados, $92,6 \%(25 / 27)$ possuir renda familiar entre 1 a 2 salários-mínimos (Tabela 1).

Tabela 1 - Características socioeconômicas, demográficas e antropométricas $(n=27)$.

\begin{tabular}{lr}
\hline Variáveis & $\mathrm{n}(\%)$ \\
\hline Sexo & \\
Masculino & $12(44,4)$ \\
Feminino & $15(55,6)$ \\
Cor/raça & \\
Branca & $3(11,1)$ \\
Parda & $9(33,3)$ \\
Amarela & $2(7,4)$ \\
Preta & $10(37)$ \\
Indígena & -
\end{tabular}

$\begin{array}{lr}\text { Não sabe } & 3(11,1) \\ \text { Escolaridade } & \\ \text { Analfabeto } & 6(22,2) \\ \text { Assina o nome } & 6(22,2) \\ \text { Ensino fundamental incompleto } & 7(25,9) \\ \text { Ensino fundamental completo } & 2(7,4) \\ \text { Ensino médio incompleto } & 2(7,4) \\ \text { Ensino médio completo } & 4(14,8) \\ \text { Situação conjugal } & \\ \text { Solteiro } & 3(11,1) \\ \text { Casado } & 14(51,9) \\ \text { Divorciado } & 2(7,4) \\ \text { Viúvo } & 8(29,6) \\ \text { Renda familiar } & \\ <1 \text { salário mínimo } & 1(3,7) \\ \text { Entre } 1 \text { a } 2 \text { salários mínimos } & 25(92,6) \\ >2 \text { salários mínimos } & 1(3,7) \\ \text { Dados antropométricos } & \\ \text { IMC } & 26,3 \pm 5,2 \\ \text { Peso } & 71,8 \pm 16,2 \\ \text { Altura } & 164,8 \pm 8,3\end{array}$

Fonte: Dados da pesquisa

Quanto aos aspectos clínicos, 22,2\% (6/27) apresentavam alguma comorbidade associada, sendo $3,7 \%(1 / 27)$ doenças oculares, $3,7 \%(1 / 27)$ doenças infecciosas, $14,8 \%$ (4/27) doença cardíaca. 81,5\% (22/27) dos participantes eram hipertensos e $81,5 \%$ (22/27) diabéticos. Um total de 16 pacientes já havia sido submetido a procedimento cirúrgico, sendo em 25,9\% (7/27) algum tipo de amputação em membros inferiores.

A tabela 2 expressa o resultado do Questionário de Claudicação Intermitente, onde a maioria, 81,5\% (22/27) não apresentou a $\mathrm{Cl}$.

Tabela 2 - Presença de Claudicação Intermitente segundo o Questionário de Claudicação de Edimburgo $(n=27)$.

\begin{tabular}{lr}
\hline Classificação & n (\%) \\
\hline Positivo/Presença de Claudicação & $2(7,4)$ \\
Negativo/Não presença de Claudicação & $22(81,5)$ \\
Incapaz de deambular & $3(11,1)$ \\
\hline
\end{tabular}

Fonte: Dados da pesquisa

Em relação ao nível de atividade física a maioria $(33,3 \% ; 9 / 27)$ relatou ser ativo. E um total de $18,5 \%$ (5/27) referiu ser muito ativo (Tabela 3 ).

Tabela 3 - Prática de atividade física segundo o IPAQ (n=27).

\begin{tabular}{lc}
\hline Classificação & $\mathbf{n}(\%)$ \\
\hline Sedentário & $5(16,1)$ \\
Ativo & $9(33,3)$ \\
Muito ativo & $5(18,5)$ \\
Irregularmente ativo A & $3(11,1)$ \\
Irregularmente ativo B & $5(18,5)$ \\
\hline
\end{tabular}

Fonte: Dados da pesquisa 
Na tabela 4 é demonstrado o escore global do SF36 em cada domínio, sendo o de maior pontuação o de "Aspectos Emocionais", com 49 $\pm 49,7$ pontos, o de menor

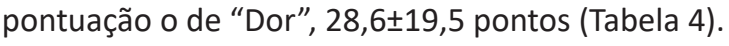

Tabela 4 - Médias e desvios padrão da qualidade de vida, segundo SF-36 $(n=27)$.

\begin{tabular}{lc}
\hline Domínio & Média (DP) \\
\hline Capacidade funcional & $28,8(22,2)$ \\
Dor & $28,6(19,5)$ \\
Limitação por aspectos físicos & $43,1(47,9)$ \\
Estado geral de saúde & $45,4(24,9)$ \\
Aspectos emocionais & $49,0(49,7)$ \\
Aspectos sociais & $37,1(26,9)$ \\
Vitalidade & $37,9(25,8)$ \\
Saúde mental & $48,7(30,3)$ \\
\hline
\end{tabular}

Fonte: Dados da pesquisa

O escore global do PSQI teve uma média de 10,4 \pm 5 e mediana de 10, indicando que os pacientes apresentam distúrbios do sono. Dos domínios analisados, a maior pontuação foi a "Duração do Sono", com média de 2,7 22,1 e mediana de 3. A menor pontuação foi a do domínio

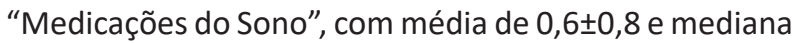
de 0 (Tabela 5).

Tabela 5 - Médias, desvios padrão e medianas dos domínios do questionário do Índice de Qualidade do Sono de PittsburghPSQI ( $n=27)$.

\begin{tabular}{lrc}
\hline Domínios PSQI & Média (DP) & Mediana \\
Qualidade do Sono & $1,4(0,8)$ & 1 \\
Latência do Sono & $1,5(1,1)$ & 2 \\
Duração do Sono & $2,7(2,1)$ & 3 \\
Eficiência do Sono & $1,3(1,2)$ & 1 \\
Distúrbios do Sono & $1,9(0,3)$ & 2 \\
Medicações do Sono & $0,6(0,8)$ & 0 \\
Sonolência & $1,8(1,7)$ & 2 \\
GLOBAL & $10,4(5,0)$ & 10 \\
\hline
\end{tabular}

Fonte: Dados da pesquisa

\section{DISCUSSÃO}

O estudo revelou uma composição amostral predominante de mulheres $(55,6 \%)$ e idosos de ambos os sexos (70\%). A maioria dos pacientes praticavam atividade física (84\%), apresentavam distúrbios no sono e repercussões negativas na qualidade de vida, principalmente relacionadas a dor e limitação física.

Acerca do Nível de Atividade Física, Alvim et al., (2018) observaram o sedentarismo como sendo de alta prevalência em pacientes com DAP (ALVIM et al., 2018). Contudo, este estudo apresenta uma amostra predominantemente classificada como ativa/muito ativa. Acredita-se que esse resultado possa ter sido influenciado por a maior parte dos pacientes $(81,5 \%)$ não apresentaram Claudicação Intermitente. Pacientes com Claudicação Intermitente apresentam maior limitação para realização de atividade física com intensidades moderadas e vigorosas (GERAGE et al., 2019), bem como, para execução das atividades de vida diária (BARBOSA; DALLE, 2008).

Por ser uma amostra composta por idosos e com comorbidades associadas, sendo hipertensão $(81,5 \%)$ e diabetes $(81,5 \%)$ as mais prevalentes, acredita-se que haja uma dificuldade maior para a prática das atividades que demandam mais tempo para serem realizadas, mas que os afazeres domésticos sejam ainda realizados. Tais afazeres colaboram, significativamente, para que os níveis de atividade física sejam considerados maiores pelos idosos (CARDOSO et al., 2008). Assim, acredita-se que a amostra tenha referido ser ativa, devido às atividades domésticas realizadas diariamente, não necessariamente por praticarem atividade física regular, já que pela falta de recursos financeiros dificultam a contratação de serviços de auxílio nestas tarefas.

A amostra estudada, em sua maioria, recebe entre um e dois salários mínimos (92,6\%) que são direcionados para alimentação, medicamentos e despesas fixas mensais. Vale ressaltar que, em algumas famílias, principalmente as residentes no interior, o idoso é a principal fonte de renda (PIGNATTI; BARSAGLINI; SENNA, 2011).

Observa-se baixa pontuação no domínio "Dor" 28,6 no questionário de qualidade de vida, sendo o domínio mais limitante. No estudo de Moreira et al. (2016), também é possível observar uma pontuação relativamente baixa $(37,5)$ nesta mesma população (MOREIRA et al., 2016), enquanto Magalhães et al. (2011), demonstra uma pontuação maior 51,4.

A dor é uma experiência sensorial desagradável e subjetiva que limita fisicamente e emocionalmente (SALLUM; GARCIA; SANCHES, 2012), podendo acarretar prejuízos consideráveis, principalmente nas relações sociais, bem como na qualidade de vida (RODRÍGUEZ et al., 2019). Sallum, Garcia e Sanches (2012) em um estudo de revisão narrativa da literatura, trazem a diminuição do sono como um complicador para a melhora do quadro álgico, em especial a dor aguda, já para Vega et al. (2019) quanto mais partes do corpo o paciente apresentar dor, maiores serão as perturbações no sono.

Apesar da amostra ser ativa, a pontuação para o domínio "vitalidade" na SF-36 pode sinalizar sensação de cansaço e descontentamento para a execução das atividades em uma boa parte do tempo. Entretanto, para não se sentir dependente do auxílio de outras pessoas, alguns indivíduos optam por realizar as atividades, mesmo cansados e com dor (RODRÍGUEZ et al., 2019).

As baixas pontuações no domínio "limitação por aspectos físicos" podem comprometer o tempo e a qualidade de execução das atividades de vida diária, mesmo que os pacientes ainda as realizem. Moreira et al. (2016), em um estudo clínico descritivo e transversal, identificaram valores baixos em relação aos "aspectos físicos" $(13,7)$ dos pacientes com DAP, devido à insatisfação por realizar tarefas ocupacionais ou diárias. 
Também foram observadas baixas pontuações nos "aspectos emocionais", "aspectos sociais" e "saúde mental", indicando estresse e insatisfação, corroborando com o estudo de Wann-Hansson et al. (2005) em que os autores relatam sobre a experiência de se viver com a DAP, destacando o fato destes pacientes se isolarem, seja por limitações físicas, pela dor ou até mesmo pela solidão por serem restritos de atividades sociais.

A amostra apresenta distúrbios no sono relacionados, principalmente, à duração. Sabanayagam e Shankar (2010), em um estudo transversal, observaram que dormir por poucos períodos oferece maiores riscos para o surgimento ou agravo das doenças. Para Watanabe et al. (2010), a curta duração do sono está associada ao risco para comorbidades como a obesidade. Nota-se que o Índice de Massa Corporal), da amostra estudada, denota sobrepeso $(25,2)$ podendo estes pacientes se tornarem obesos futuramente. Além disso, segundo Vasconcelos et al. (2013), os indivíduos com um IMC que revele sobrepeso, podem estar propensos á apresentarem alterações no sono.

Silva et al. (2011), apontaram que além da qualidade ruim do sono ser um fator predisponente da obesidade, repercute negativamente nos aspectos emocionais. Apesar de os "aspectos emocionais" terem sido o domínio mais pontuado da qualidade de vida (49), esse valor ainda corresponde à metade da pontuação máxima a ser obtida, podendo assim indicar desânimos em alguns momentos. Acredita-se que a dor pode ter sido um fator determinante para o distúrbio no sono da amostra, como demonstrado no trabalho de Vega et al. (2019) e no trabalho de Sallum, Garcia e Sanches (2012).

São limitações deste estudo a possibilidade de viés de memória, uma vez que os pacientes responderam aos questionários com base em sua rotina antes da hospitalização, bem como a possível omissão de informações por parte dos pacientes no que se refere ao estado de saúde.

\section{CONCLUSÃO}

A DAP acometeu principalmente indivíduos idosos, acarretou diminuição na qualidade de vida e sugere indícios de distúrbios do sono. Neste estudo, o fato de os pacientes relatarem serem ativos antes da internação, pode não se relacionar diretamente com a prática de exercícios físicos regulares, e sim de atividades de vida diária, uma vez que o IPAQ considera essas atividades para pontuação no questionário. Neste contexto, os profissionais de saúde ao assistirem esses indivíduos, devem avaliar não somente aspectos imediatos, como a dor e as condições do membro acometido pela DAP, mas também, incluir uma avaliação acerca da qualidade do sono e vida. Logo, a adoção de hábitos de vida saudáveis, como a prática regular de exercício físico, bem como investimentos na promoção e prevenção de agravos à saúde, pode promover melhor qualidade de vida e do sono para estes pacientes.

\section{REFERÊNCIAS}

ALVIM, R. O. et al. Prevalence of peripheral artery disease and associated risk factors in a brazilian rural population: the baependi heart study. Int. J. Cardiovasc. Sci., Rio de Janeiro, v. 31, n. 4, p. 405-413, June 2018.

ARAGÃO, J. A. et al. Qualidade de vida em pacientes com doença arterial periférica. J. Vasc. Bras., Rio de Janeiro, v. 17, n. 2, p. 117-121, jun. 2018.

BARBOSA, E. C.; DALLE, R.D.M. Benefícios do condicionamento físico como tratamento da claudicação intermitente Benefits of physical conditioning in the treatment of intermittent claudication. Acta Fisiátrica, São Paulo, v.15, n. 3, p. 192-194, Sept. 2008.

BARROS, M. B. A. et al. Quality of sleep, health and well-being in a population-based study. Rev. Saúde Públ., São Paulo, v. 53, n. 82, p. 1-12, Sept. 2019.

CARDOSO, A. S. et al. Comparação do nível de atividade física em relação ao gênero de idosos participantes de grupos de convivência. Revista Brasileira de Ciências do Envelhecimento Humano, Passo fundo, v. 5, n. 1, p. 9-18, jun. 2008.

FARAH, B. Q. et al. Effects of clustered comorbid conditions on walking capacity in patients with peripheral artery disease. Ann. Vasc. Surg., Detroit, v. 28, n. 2, p. 279-283, Feb. 2014.

FLECK, M. P. A. O instrumento de avaliação de qualidade de vida da Organização Mundial da Saúde (WHOQOL-100): características e perspectivas. Ciênc. Saúde Colet., Rio de Janeiro, v. 5, n. 1, p. 33-38, mar. 2000.

GERAGE, A. M. et al. Níveis de atividade física em pacientes com doença arterial periférica. Arq. Bras. Cardiol., São Paulo, v. 113, n. 3, p. 410416, set. 2019.

JAMES, S. L et al. GBD 2017 Disease and Injury Prevalence. Global, regional, and national incidence, prevalence, and years lived with disability for 354 diseases and injuries for 195 countries and territories, 1990-2017: a systematic analysis for the Global Burden of Disease Study 2017. Lancet, London, v. 392, n.10159, p. 1789-1858, Nov. 2018.

KENGNE, A. P.; ECHOUFFO-TCHEUGUI, J. B. Differential burden of pheripheral artery disease. Lancet, London, v. 7, n. 8, p. 980-981, Ago. 2019.

LAGUARDIA, J. et al. Dados normativos brasileiros do questionário Short Form-36 versão 2. Rev. Bras. Epidemiol., São Paulo, v. 16, n. 4, p. 889-897, dez. 2013.

MAGALHÃES, S. et al. Peripheral arterial disease: exclusion criteria for exercise training?. Revista da Sociedade Portuguesa de Medicina Física e de Reabilitação, [s.I], v. 20, n. 1, p. 1-6, 2011.

MAKDISSE, M. et al. Versão em português, adaptação transcultural e validação do Questionário de Claudicação de Edimburgo. Arq. Bras. Cardiol., São Paulo, v. 88, n. 5, p. 501-506, maio 2007.

MATSUDO, S. et al. Questionário Internacional De Atividade Física (Ipaq): Estudo De Validade E Reprodutibilidade No Brasil. Rev. Bras. Ativ. Fís. Saúde, Londrina, v. 6, n. 2, p. 5-18, 2001.

MOREIRA, M. M. R. et al. Qualidade de vida e capacidade funcional em pacientes com úlcera arterial. Av. Enferm., Bogotá, v. 34, n. 2, p. 170-180, dez. 2016.

MUIR, R. L. Peripheral arterial disease: Pathophysiology, riskfactors, diagnosis, treatment, andprevention. J. Vasc. Nurs., Norwood v. 27, n. 2, p. 26-30, June 2009 .

PASCOTTO, A. C.; SANTOS, B. R. M. Avaliação da qualidade do sono em estudantes de ciências da saúde assessing sleep quality in health occupations students. J. Health Sci. Inst., São Paulo, v.31, n.3, p. 306310, Sept. 2013. 
PIGNATTI, M. G.; BARSAGLINI, R. A; SENNA, G.D. Envelhecimento e rede de apoio social em território rural do Pantanal mato-grossense. Physis, Rio de Janeiro, v. 21, n. 4, p. 1469-1491, Oct. 2011.

RODRÍGUEZ, I. et al. Living with chronic pain: A qualitative study of the daily life of older people with chronic pain in Chile. Pain Res. Manag., London, v. 2019, p. 1-9, Apr. 2019.

SABANAYAGAM, C.; SHANKAR, A. Sleep duration and cardiovascular disease: results from the national health interview survey. Sleep, Oxford, v. 13, n. 2, p. 145-150, Aug. 2012.

SALLUM, A. M. C.; GARCIA, D. M.; SANCHES, M. Dor aguda e crônica: revisão narrativa da literatura. Acta Paul. Enferm., São Paulo, v. 25, n. 1, p. 150-154, maio 2012.

SHABANI VARAKI, E. et al. Peripheral vascular disease assessment in the lower limb: a review of current and emerging non-invasive diagnostic methods. Biomed. Eng. Online, London, v. 17, n. 1, p. 1-27, May 2018.

SILVA, R. C. G.; CONSOLIM-COLOMBO, F. M. Aspectos relevantes para identificação da claudicação intermitente. Acta Paul. Enferm., São Paulo, v.24, n.3, p. 426-429, 2011.

VASCONCELOS, H. C. A. Correlação entre indicadores antropométricos e a qualidade do sono de universitários brasileiros. Rev. Esc. Enferm. USP, São Paulo, v. 47, n. 4. p. 859-859, Ago. 2013.

VAZ, C. et al. Doença arterial periférica e qualidade de vida. Angiol. Cir. Vasc., Rio de Janeiro, v. 9, n. 1, p. 17-23, Mar. 2013.

VEGA, R. DE L. A. et al. Sleep disturbance in individuals with physical disabilities and chronic pain: the role of physical, emotional and cognitive factors. Dis. Health J., Amsterdam v. 12, n. 4, p. 588-593, Out. 2019.

VIRANI, S.S. et al. Heart disease and stroke statistics-2020 update a report from the American heart association. Circulation, Dallas, v. 141, n. 9, p.1-458, Jan. 2020.

WANN-HANSSON, C. et al. Patients' experiences of living with peripheral arterial disease awaiting intervention: a qualitative study. Int. J. Nurs. Stud., Oxford, v. 42, n. 8, p. 851-862, Nov. 2005.

WATANABE, M. et al. Association of short sleep duration with weight gain and obesity at 1-year follow-up: a large-scale prospective study. Sleep, Oxford, v. 33, n. 2, p. 161-167, Feb. 2010.

WU, A. et al. Lower extremity peripheral artery disease and quality of life among older individuals in the community. J. Am. Heart Assoc., London, v. 6, n. 1, p. 1-13 Jan. 2017.

Submetido em: $16 / 04 / 2020$

Aceito em: 25/01/2021 